\title{
Revista Brasileira de Medicina de Familia e Comunidade
}

\section{Repercussões tardias da acupuntura e moxabustão na qualidade de vida e dor na síndrome pós-pólio}

Celso Antonio de Souza Mello, Gislaine Cristina Abe,Paulo Eduardo Ramos, Sissy Veloso Fontes, Bruna Terumi Sato Yonamine, AbrahćoJuviniano Quadros, Beny Schmidt, Acary Souza Bulle Oliveira

${ }^{1}$ Setor de Investigação em Doenças Neuromusculares da Disciplina de Neurologia Clínica do Departamento de Neurologia e Neurocirurgia da Universidade Federal de São Paulo

E-mails: celsomellinho@hotmail.com ${ }^{1}$, gislaineabe@hotmail.com ${ }^{1}$, paulo_ramos09@hotmail.com ${ }^{1}$, sissyfontes@gmail.com ${ }^{1}$, brunayonamine@gmail.com ${ }^{1}$, aajquadros@gmail.com ${ }^{1}$, benyschmidt@gmail.com ${ }^{1}$, acary.bulle@unifesp.br

Introdução:A síndrome pós-poliomielite (SPP) é um quadro que aparece em pessoas sobreviventes da poliomielite aguda, que após um período de estabilidade começam a sentir novos sinais e sintomas, dentre eles, fraqueza, fadiga, dor muscular e a dor articular, sendo um quadro progressivo, e até o momento, não apresenta tratamento específico. O objetivo deste estudo foi verificar por quanto tempo persistem os efeitos do tratamento de acupuntura e moxabustão em relação à qualidade de vida e dor em pacientes com SPP com queixa de dor. Método: oito pacientes foram acompanhados após o término do tratamento e mensalmente durante seis meses consecutivos, sendo 6 mulheres e 2 homens, com idades entre 40 e 58 anos, que foram submetidos a 20 sessões de acupuntura de 30 minutos cada, duas vezes por semana, durante 2 meses e meio. Para a avaliação foram aplicados a escala visual analógica (EVA) para dor, o questionário McGill para dor e o instrumento abreviado de avaliação da qualidade de vida - Whoqol-bref. Os resultados não foram comparados com os do início do tratamento com acupuntura, mas todos os pacientes relatavam melhora subjetiva dos sintomas. Houve diferença estatisticamente significativa somente em EVA, mostrando retorno da dor após 6 meses do término do tratamento. Houve tendência a diferenças significantes em McGill, e diferenças consideradas não significantes no Whoqol-bref indicando manutenção da qualidade de vida, porém os pacientes relatavam, subjetivamente, piora dos sintomas. Conclusões:1. A dor teve aumento estatisticamente significativo após 6 meses de acompanhamento na Escala EVA-dor; 2. A qualidade de vida não apresentou modificação nesse período; 3 . O acompanhamento após terapia dos pacientes com doenças neuromusculares é extremamente importante para se observar a interferência da técnica na história natural da doença.

Palavras-chave:Síndrome pós-poliomielite.Medicina Tradicional Chinesa.Acupuntura. Dor. Qualidade de vida. 